Cipango Cahiers d'études japonaises

17 | 2010

La péninsule retrouvée

\title{
Regards sur la métaphore, entre Orient et Occident, Cécile SAKAI et Daniel STRUVE (dir.)
}

\author{
Marianne Simon-Oikawa
}

\section{(2) OpenEdition}

\section{Journals}

\section{Édition électronique}

URL : https://journals.openedition.org/cipango/1140

DOI : $10.4000 /$ cipango. 1140

ISSN : 2260-7706

\section{Éditeur}

INALCO

Édition imprimée

Date de publication : 30 juin 2010

Pagination : 262-268

ISBN : 978-2-85831-195-8

ISSN : $1164-5857$

\section{Référence électronique}

Marianne Simon-Oikawa, «Regards sur la métaphore, entre Orient et Occident, Cécile sakal et Daniel Struve (dir.) », Cipango [En ligne], 17 | 2010, mis en ligne le 16 novembre 2012, consulté le 30 juin 2021. URL : http://journals.openedition.org/cipango/1140; DOI : https://doi.org/10.4000/cipango.1140

Ce document a été généré automatiquement le 30 juin 2021.

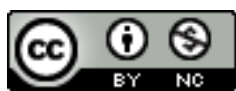

Cipango est mis à disposition selon les termes de la Licence Creative Commons Attribution - Pas d'Utilisation Commerciale 4.0 International. 


\title{
Regards sur la métaphore, entre Orient et Occident, Cécile SAKAI et Daniel STRUVE (dir.)
}

\author{
Marianne Simon-Oikawa
}

\section{RÉFÉRENCE}

Cécile SAKAI et Daniel STRUVE (dir.), Regards sur la métaphore, entre Orient et Occident, Arles, Philippe Picquier, 2008, 322 pages. ISBN 978-2-8097-0084-8

1 L'Extrême-Orient n'est plus une terre lointaine et incompréhensible. Il fait aujourd'hui partie intégrante, virtuellement du moins, de l'horizon culturel commun. Il est à ce titre de plus en plus souvent convoqué dans le cadre de problématiques visant à comprendre comment est envisagée, c'est-à-dire nommée et construite, dans plusieurs cultures, une question essentielle. C'est justement ce à quoi s'attache aussi l'ouvrage dirigé par Cécile Sakai et Daniel Struve, consacré à la métaphore. Partant du constat que l'orientaliste perçoit et interprète son objet d'étude à travers un prisme particulier, qu'il doit interroger, l'introduction pose la nécessité d'élaborer « une méthode qui intègre une dimension comparatiste et fait place, sans les confondre ni les rabattre l'un sur l'autre, à chacun des systèmes de pensée et de référence dont elle est tributaire " (p.9). Les quinze articles qui suivent reprennent à leur compte ce programme, et tentent chacun, à partir d'exemples empruntés à l'Inde, à la Chine et au Japon, d'« introduire des cas nouveaux, présenter des configurations différentes de celles que l'on peut observer en Occident, tracer des lignes et suggérer des rapprochements " (p. 12).

2 Dans le champ littéraire, on pouvait difficilement trouver objet moins consensuel, et par là plus stimulant, que la métaphore. Les auteurs le rappellent d'entrée de jeu, « elle a donné lieu à des débats innombrables, parfois inextricables, à cause de la diversité des approches possibles et de la difficulté même de s'entendre sur une définition» (p. 9). 
Entretient-elle avec la comparaison une différence de nature ou simplement de degré ? Quels sont ses liens avec la métonymie, le symbole, l'allégorie? Où se situe la frontière entre «métaphore vive» (Ricœur) ou d'«invention», et stéréotype? Les analyses occidentales, à commencer par celles des rhétoriciens français (Les Figures du discours de Fontanier, publié en 1968 chez Flammarion, est souvent cité par les auteurs du volume), divergent sur tous ces points. En Extrême-Orient, la métaphore est aussi très présente mais sous divers noms, et ses formes ne sont pas toujours perçues comme concurrentes d'autres figures. Son analyse doit en outre tenir compte d'une histoire spécifique, dans laquelle la découverte de l'Occident a joué un rôle déterminant.

3 C'est un linguiste, Vincent Nyckees, qui ouvre la réflexion en traitant du « sens figuré en langue et en discours", et plus particulièrement des "sources linguistiques de l'énonciation métaphorique ». Sa critique des approches sémantiques classiques de la métaphore le conduit à souligner la " continuité entre métaphores vives et métaphores éteintes" (p. 29), et à proposer de nouveaux modèles d'analyse des énoncés métaphoriques. Les contributions suivantes s'attachent à cerner les formes et les usages de la métaphore dans différents corpus extrême-orientaux. La première partie, consacrée à l'Inde et au Japon, s'ouvre sur les textes bouddhiques, évidemment fondateurs, qu'analyse Frédéric Girard. «La prédication attribuée au Buddha s'exprime volontiers à l'aide de métaphores, d'allégories et de symboles ", rappelle-t-il (p. 35). Dans la mesure où la vérité religieuse échappe à l'argumentation rationnelle, on comprend qu'elle ait souvent recours à l'expression indirecte, en particulier à la métaphore. Le statut spécifique de cette dernière est cependant difficile à cerner, ne serait-ce que parce que "le terme même de métaphore, synonyme de symbole et d'allégorie, est rendu par plusieurs termes en sino-japonais, qui néanmoins se recoupent » (p. 40). Girard montre toutefois que le bouddhisme n'a pas été indifférent à la réflexion sur la métaphore en tant que telle, et qu'il distingue par exemple " les métaphores d'emprunt, nominales ou fictives (keyu) et les métaphores réelles (jitsuyu)» (p. 42).

4 C'est ce même décalage entre l'objet et ses dénominations qu'observe Stéphane Feuillas dans le cas de la littérature chinoise, qu'il aborde à partir de la poésie du lettré Su Shi ( $\mathrm{xI}^{\mathrm{e}}$ siècle). Il remarque que si le rapprochement en général et la métaphore en particulier constituent chez lui une dynamique fondamentale, l'œuvre résiste aux découpages de la rhétorique occidentale : "Qu'un marqueur de la comparaison soit présent ou non, on serait en peine de distinguer avec profit la synecdoque de la métonymie, l'hypallage de l'allégorie. Tout semble possible dans tout» (p. 58). Dans le domaine littéraire encore, c'est une lecture lacanienne d'une métaphore particulière, celle de la pierre, que propose ensuite Rainier Lanselle. Dans le Rêve du pavillon rouge, d'abord intitulé par son auteur Shitou ji, soit L'Histoire de la pierre ou Mémoires de la pierre, la pierre joue selon lui «comme une métaphore inouïe, d'une remarquable valeur symbolique, de ce qui arrive au sujet humain, c'est-à-dire au sujet de la langue » (p. 87). La naissance du héros, venu au monde avec dans la bouche un jade, annoncerait ainsi l'issue du roman, en même temps qu'elle justifierait le recours de l'auteur à l'expression indirecte: "Métaphore de la pierre primordiale, le jade devient la métaphore du sujet parlant, c'est-à-dire voué à l'incommunicabilité et au recours au détour métaphorique» (p.103). Le point de vue psychanalytique, "très inhabituel, pour ne pas dire sans précédent, dans l'approche des textes de la Chine classique " (p. 83), démontre ici brillamment sa pertinence. La métaphore n'est certes pas l'apanage de la littérature. L'utilisation des métaphores dans le discours esthétique, en 
particulier dans les textes techniques concernant la calligraphie et la musique, est fréquente, comme le montre Véronique Journeau-Alexandre. "Une goutte de rosée descend obliquement vers la droite ", dit une stèle de Wang Xizhi pour décrire le point calligraphique (p. 109). "C'est un oiseau à une seule patte dont l'esprit connaît la pluie ", lit-on dans un manuel de cithare (p.113). Mais ces métaphores, récurrentes dans les ouvrages techniques, le sont plus encore dans les poèmes qui évoquent une émotion esthétique ressentie devant une œuvre picturale ou musicale, certaines pouvant d'ailleurs s'appliquer aux deux arts.

On est donc surpris de constater que la fréquence de la métaphore dans le discours religieux, littéraire ou esthétique, n'a pas eu pour corollaire en Chine le développement d'une réflexion très poussée sur la métaphore elle-même. Chantal Chen-Andro montre au contraire que la principale caractéristique de la terminologie critique dans ce domaine est le flou. La concurrence entre plusieurs termes (yinyu, xingxiang, yingxiang), la polyvalence du mot biyu, qui désigne la comparaison mais peut «s'employer aussi pour la métaphore ou l'allégorie»(p.132), est patente. Mais elle ne saurait se comprendre comme l'expression d'un simple manque de rigueur. Pris entre une tradition nationale qu'ils connaissaient mal, et des lectures pour eux toutes nouvelles (les poètes symbolistes français, Mallarmé), les critiques chinois de la première moitié $\mathrm{du} \mathrm{xx}^{\mathrm{e}}$ siècle n'avaient peut-être pas d'autre choix que de recourir à un bricolage terminologique, dont l'histoire est inséparable de celle de la littérature chinoise dans son ensemble. C'est encore le bricolage, d'ailleurs, qui semble caractériser l'usage de la métaphore dans le processus néologique du chinois contemporain. Analysant un corpus de mots d'emprunt, Qi Chong y repère deux types de métaphores, « de similitude » et "de proximité ", dont le glossaire, placé en fin d'article, fournit quelques exemples délicieux. On apprécie shengna, de l'anglais sonar, écrit avec les caractères qui signifient "son-réception" (métaphore de similitude), mais plus encore xiangbo, "parfuméevague ", qui traduit, par une métaphore de proximité cette fois-ci, l'anglais shampoo...

6 Pas plus qu'en Chine, la terminologie occidentale ne semble au Japon, qui fait l'objet de la deuxième partie de l'ouvrage, pertinente pour analyser l'ensemble des procédés rhétoriques observés. La métaphore y est non seulement désignée par plusieurs termes, mais cohabite aussi avec d'autres procédés dont l'analyse doit tenir compte.

7 Terada Sumie observe ainsi, à propos des poèmes d'Ôtomo no Yakamochi (VIII siècle) : « Nous avons de sérieuses raisons de douter de l'efficacité, pour cerner le mécanisme de la poésie antique japonaise, de l'approche fondée sur la distinction entre la métaphore (axe paradigmatique) et la métonymie (axe syntagmatique)»(p. 179). Le mot "métaphore» lui-même (in.yu) est-il pertinent pour l'analyse de la poésie de Yakamochi ? Sur un corpus de 450 poèmes, seuls 3 contiennent une métaphore au sens strict du terme (p. 180). Le rapprochement de réalités hétérogènes y est récurrent mais il emprunte d'autres voies, en particulier celle du mitate, qui repose sur un «jeu des ressemblances » (p. 185). Michel Vieillard-Baron dresse un constat similaire à propos des poèmes d'amour du Kokin wakashû (Recueil de poèmes anciens et modernes, compilé au début $d u \mathrm{x}^{\mathrm{e}}$ siècle). «Sur les 360 poèmes d'amour, 35 contiennent une métaphore, soit moins d'un waka sur $10 »$ (p. 195), signe non pas d'un refus de la métaphore elle-même, mais au contraire d'une "utilisation plus variée des nombreuses figures auxquelles pouvaient avoir recours les poètes » (p. 209). Dans le Japon ancien, il est en tout cas une métaphore récurrente, en prose comme en poésie, qu'étudie Claire-Akiko Brisset : celle du végétal pour renvoyer à l'écriture. S'attachant plus particulièrement à un style 
appelé ashide, qui désigne des "caractères en forme de roseau " (p. 216), Brisset rappelle l'histoire du mot, et la variété de ses acceptions : susceptibles d'apparaître sur différents supports, les ashide ont pu «tout d'abord orner les traînes des dames de la cour impériale, servir à la transcription de poèmes sur les objets les plus divers ou encore agrémenter des peintures ou des sous-motifs de calligraphies » (p. 221). Par un intéressant phénomène de renversement, ces signes hybrides, à la fois signes d'écriture et motifs picturaux, purent même servir de pivot à une " transmutation du paysage en page d'écriture » (p. 223), un spectacle dans un jardin pouvant par exemple évoquer, dans l'esprit des spectateurs, une page de ashide.

Passant du Japon classique à l'époque prémoderne, Daniel Struve s'attache à la figure attendue de Bashô, non tant pour souligner son art bien connu de la brièveté, que pour analyser la manière dont s'enchaînent, dans les haikai composés avec ses disciples, les différentes séquences de chacun. Le « déplacement " (utsuri), « qui évite un rapport de contiguïté trop étroit ", joue un rôle essentiel dans le déroulement de l'ensemble du poème. Mais quel terme de rhétorique pourrait désigner ce type d'expression indirecte, particulièrement favorable à l'apparition de métaphores, qui caractérise la poésie de Bashô : nioizuke? "Enchaînement par le parfum ", propose Struve (p. 236). Voilà qui ouvre de nouvelles perspectives à la rhétorique occidentale. Ne pourrait-on trouver aussi chez tel ou tel de nos symbolistes, des « enchaînements par le parfum ", reposant sur un certain air de familiarité des choses entre elles, unies par une sorte d'odeur commune ? Bashô était évidemment loin de se poser de telles questions. Les Japonais ont réfléchi à la traduction du mot « métaphore » dans leur langue, mais bien plus tard. C'est au cours de l'ère Meiji, avec l'ouverture du pays à l'Occident, que la réflexion sur la métaphore comme figure rhétorique s'est développée dans le discours critique. Dans son article, qu'il est intéressant de mettre en regard avec celui de Chantal Chen-Andro, Massimiliano Tomasi montre l'évolution des travaux de rhétorique qui se développèrent à partir des années 1870 : ils empruntèrent d'abord leurs exemples à la seule littérature classique, et ne vinrent que tardivement à la littérature contemporaine. "C'est seulement dans les toutes dernières années de Meiji que la rhétorique, et avec elle les figures de rhétorique, commencèrent à être traitées comme étant des composants essentiels du matériau linguistique contemporain, et non plus comme de simples vestiges du passé » (p. 259).

9 Si les métaphores étaient déjà très présentes dans le roman de Meiji, elles pullulent dans la poésie d'après-guerre, que Makiko Andro-Ueda caractérise même comme une "poésie de la métaphore" (p.264). L'analyse de quelques poèmes des années cinquante, choisis dans l'œuvre de poètes comme Ayukawa, Yoshioka, Tanikawa ou encore Sasaki, illustre l'importance de ce procédé, qui atteint « son point de saturation dans les années 1970-1980 ", chez Arakawa ou Itô. C'est sur le début des années quatrevingt que s'attarde lui aussi Emmanuel Lozerand, avec son analyse d'un recueil de nouvelles de Mukôda Kuniko, Omoide toranpu (Les Cartes du souvenir), dans lequel prolifèrent les images (métaphores mais aussi comparaisons, métonymies, allégories). Dans ces textes qui explorent le mensonge (uso) sous toutes ses formes, Lozerand voit l'expression d'un paradoxe, celui de l'association d'une trame narrative « souvent orientée vers la critique ou la dénonciation des images » et d'un style au contraire « abusant de leur emploi » (p. 298).

10 Que les textes critiques des romanciers et des poètes accordent peu de place à la métaphore ne signifie donc pas que celle-ci soit absente de leur écriture. Les 
métaphores sont partout. On les trouve jusque dans les titres d'un grand nombre de romans. Mais ont-elles toutes la même densité, la même fraîcheur ? Sont-elles toutes des métaphores d'«invention»? L'article de Cécile Sakai apporte à l'ouvrage une conclusion stimulante, en abordant de front la question du rapport entre métaphore et stéréotype. Loin de rejeter ce dernier dans la sphère de la littérature populaire, qui en fait assurément grand usage, l'auteur montre au contraire la multiplicité des liens entre " métaphore conventionnelle ( (Lakoff et Johnson) et "métaphore d'invention » dans le roman japonais contemporain (p. 302). Que des métaphores d'invention se muent en lieux communs ne signifie pas nécessairement un appauvrissement esthétique. Le phénomène, qui "déconstruit les cadres ", constitue plutôt à ses yeux un signe de modernité, et invite à formuler une hypothèse que l'on voudrait voir reprise et développée en une autre occasion: «la distance qui sépare métaphore d'invention et métaphore conventionnelle ne serait pas, au Japon, aussi importante que dans les cultures occidentales » (p. 317).

11 Parti du point de vue de l'orientaliste sur son objet d'étude, le volume se referme ainsi provisoirement sur une comparaison entre Orient et Occident. Il ne l'a jamais, à vrai dire, quittée. On a été sensible, tout au long de l'ouvrage, aux échos relevés par les auteurs entre leur corpus d'étude et certaines références occidentales qui pouvaient en sembler a priori très éloignées. Le rapprochement que fait Stéphane Feuillas entre « Le Mont du Dragon ennuagé » de Su Shi et «Mystique » de Rimbaud est indéniablement stimulant (p.63). Plus audacieusement encore, Véronique Journeau-Alexandre montre que la phrase, jugée irrecevable par Chomsky, «les idées vertes, incolores, dorment furieusement", "peut être considérée comme une métaphore qui participe d'un imaginaire partagé en Chine et évoque la mante religieuse, tapie immobile à l'affût de sa proie, modèle pour la réalisation d'un geste musical, d'une figuration picturale, et même pour la boxe chinoise » (p. 123-124).

Quittant le terrain retenu pour l'analyse menée ici, on pourrait peut-être pousser plus loin encore la réflexion sur la métaphore en la mettant en relation avec l'écriture idéographique elle-même, sur laquelle, de manière surprenante, peu d'articles s'étendent. Qi Chong mentionne bien ces «jeux graphiques» qui entrent parfois en compte dans la conception d'emprunts, mais pour affirmer immédiatement qu'ils " sont très rares et ne peuvent être interprétés comme des métaphores » (p. 163). Qu'en est-il de la poésie ? L'exemple que Qi Chong donne, la graphie du mot «pingpang» (de l'anglais ping-pong) nous évoque justement un poème très connu d'Eugen Gomringer intitulé Ping-pong, purement alphabétique, mais qui cherche lui aussi à représenter « le mouvement du rebond de la balle de ping-pong » (p. 163). Le mot « métaphore » est-il à ce point impropre à caractériser une configuration de ce type ? De manière générale, l'ouvrage laisse de côté, ce n'était pas son objet, les interprétations métaphoriques que l'Occident a pu faire de l'écriture chinoise et japonaise. Il y a là pourtant un corpus à la fois poétique et critique intéressant, et qui mériterait, dans un autre cadre, d'être mis en perspective par rapport à des travaux orientalistes. Ernest Fenollosa (1853-1908), dans son livre The Chinese Written Character as a Medium for Poetry, publié par Ezra Pound en 1920, n'affirme-t-il pas par exemple que l'idéogramme «bears its metaphor on its face »? Une telle interprétation, de nature à alimenter le rêve d'une écriture métaphorique au sens le plus radical du terme, ne peut qu'inviter à poursuivre la réflexion vigoureusement engagée ici sur la métaphore en Orient et en Occident. 